\title{
Médiévales
}

Langues, Textes, Histoire

71 | automne 2016

Conflits et concurrence de normes

\section{Conflits et concurrence de normes}

Conflicts and competition of norms

\section{Véronique Beaulande-Barraud et Elsa Marmursztejn}

\section{(2) OpenEdition}

Journals

Édition électronique

URL : https://journals.openedition.org/medievales/7875

DOI : $10.4000 /$ medievales. 7875

ISSN : $1777-5892$

Éditeur

Presses universitaires de Vincennes

Édition imprimée

Date de publication : 20 novembre 2016

Pagination : 5-12

ISBN : 978-2-84292-565-9

ISSN : 0751-2708

Référence électronique

Véronique Beaulande-Barraud et Elsa Marmursztejn, «Conflits et concurrence de normes », Médiévales [En ligne], 71 | automne 2016, mis en ligne le 20 novembre 2018, consulté le 24 avril 2022. URL : http:// journals.openedition.org/medievales/7875; DOI : https://doi.org/10.4000/medievales.7875 


\author{
Véronique Beaulande-Barraud et Elsa Marmursztejn
}

\title{
Conflits et concurrence de normes
}

Les cinq études consacrées aux «Conflits de normes » réunies dans ce numéro thématique de la revue Médiévales prolongent la perspective ouverte par les travaux accueillis en 2008 et 2009 à 1'Université de Reims, puis recueillis dans le volume publié en 2012 sur le thème de «La fabrique de la norme ${ }^{1} \gg$. Il s'agissait alors de dresser un état des lieux et des modes de production de la norme, en des siècles caractérisés par la fragmentation des droits et la pluralité des instances normatives, en tenant compte à la fois du contexte large et des conditions spécifiques de cette production. Ces études manifestent la productivité de la «fabrique » autant que sa capacité à mettre en œuvre des questionnements historiques issus d'horizons divers et situés au carrefour d'évolutions historiographiques récentes. Le rapprochement de l'histoire et du droit ${ }^{2}$, d'une part, l'élargissement du concept de norme au-delà du droit ${ }^{3}$, d'autre part, justifiaient que soient ressaisis, en divers champs et spécialités de l'histoire et dans le spectre le plus large possible des significations de la «norme », des codes de comportement multiples. L'éventail normatif est large, des prescriptions codifiées aux règles de conformité non écrites, mais prégnantes et porteuses de contraintes réelles : de l'Université de Paris à la cour de Versailles, des lois barbares aux officialités, de la casuistique au procès, la norme, ou plutôt les normes, se construisent, s'élaborent dans des laboratoires divers, dans la construction discursive la plus savante comme dans le «bricolage » entre acteurs divers.

1. V. Beaulande, J. Claustre, E. Marmursztejn éd., La Fabrique de la norme. Lieux et modes de production des normes au Moyen Âge et à l'époque moderne, Rennes, 2012.

2. Cf. « Droit, histoire, sciences sociales », Annales ESC, 47/6 (1992) ; « Histoire et droit », Annales HSS, 57/6 (2002).

3. $C f$. C. Gauvard, A. Boureau, R. Jacob, C. De Miramon, " Les normes », dans J.-C. Schmitt et O.-G. OexLe éd., Les Tendances actuelles de l'histoire du Moyen Âge en France et en Allemagne, Paris, 2002, p. 461-492. 
Suivant l'optique pragmatique qui a orienté nos précédents travaux, ce sont toujours les processus d'élaboration des normes qui sont visés, mais ils le sont ici sous l'angle spécifique des conflits survenus ou entretenus entre des normes de sources et de types différents - conflits inévitables dans un contexte de pluralité des instances normatives, pluralité non hiérarchisée dans nombre de domaines. À l'encontre de l'idée de l'autonomie du droit et plus généralement de l'autorité déterminante des normes, on a voulu considérer ce qui leur était opposable. L'objectif n'était pas, toutefois, d'observer les distorsions entre des « normes » et des «pratiques » conçues comme des abstractions autonomes ou étanches. Il ne s'agissait pas simplement de confirmer, s'il en était besoin, l'hypothèse désormais érigée au rang d'opinion commune qui veut que le jeu social prime et que la norme soit le fruit de négociations et d'adaptations. L'enjeu consistait à envisager les processus de production des normes sous l'angle de la tension, de la concurrence ou de l'affrontement entre différents types d'instances normatives, mais aussi entre des systèmes de références qui n'avaient habituellement pas besoin d'être spécifiés et que ces moments de crise révèlent ou éclairent, de même qu'ils permettent d'observer les techniques à l'œuvre et les tactiques déployées. Un premier dossier a déjà été publié sur cette thématique, courant, comme l'ouvrage cité précédemment, du haut Moyen Âge à la fin de l'époque moderne ${ }^{4}$. Pour celui présenté ici, le resserrement chronologique sur les siècles médiévaux a été privilégié, permettant d'appréhender ces tensions dans un contexte culturel plus homogène.

Sans disparaître, la question de savoir où et comment on a produit des normes s'efface ainsi au profit d'un examen des types de conflits entre instances et entre sources normatives auxquels la production des normes a pu donner lieu, ainsi que des résultats auxquels ces conflits ont abouti. La prise en compte de la diversité des énoncés normatifs, des modalités de conflits et des dynamiques de leur résolution fait alterner, autant que possible, une réflexion théorique sur les conflits de normes et le traitement de situations particulières, de cas concrets de conflits observables au sein de différentes «fabriques » : juridique, judiciaire, religieuse, théologique... La place faite aux acteurs est un autre principe directeur du programme fixé. De fait, comme on le verra, les protagonistes des conflits envisagés par les différentes contributrices ne sont pas gouvernés par des normes qui les dépasseraient et dont ils se borneraient à enregistrer le degré d'autorité ou le rang hiérarchique. Aussi les acteurs de ces conflits de normes n'ont-ils rien de fantomatique. Leur qualité, leur engagement dans les conflits, leurs rapports de forces, leurs choix et leurs pratiques importent.

4. V. Beaulande-Barraud, E. Marmursztejn, Y. Philippe éd., « Conflits et concurrences de normes au Moyen Âge et à l'époque moderne », Savoirs en prisme, 3 (2014). La vie des normes : http://savoirsenprisme.wordpress.com/numeros/n03-2014/. 
Les travaux réunis accomplissent ce programme tout en rendant compte des recherches en cours, des recherches récentes ou $\mathrm{du}$ prolongement des recherches de leurs auteures ${ }^{5}$. Parmi ceux-ci, la question du gouvernement de l'Église et des tensions entre des impératifs potentiellement contradictoires, au sein même de la vie ecclésiale - entre pastorale, miséricorde, institutionnalisation croissante dans un cadre juridique fermement établi, exercice d'un pouvoir de coercition pour défendre l'Église et la loi divine... -, a notamment été renouvelée. Deux des textes s'insèrent dans cette problématique, montrant qu'il ne s'agit ni d'une opposition simple entre normes et pratiques, ni d'arguties de clercs sans conséquence pour la vie des fidèles. Les débats sur le mariage notamment ne sauraient être compris uniquement à l'aune d'une inadéquation des règles canoniques aux normes sociales et d'un conflit pour imposer les premières aux secondes. Carole Avignon montre comment canonistes et théologiens ont pensé la clandestinité du lien, qui crée un mariage valide mais illicite, et comment, selon le référent normatif qui l'aborde - et notamment selon qu'on se place au for de la conscience ou au for judiciaire -, l'appréciation de l'acte, peccamineux mais sacramentel, varie jusqu'à la fin du Moyen Âge. Sur un sujet aussi fondamental dans le christianisme romain que la confession, Véronique Beaulande-Barraud, par le prisme des « cas réservés », examine les contradictions entre principes canoniques savants, discours épiscopal, normes de la «cure des âmes ». C'est aussi l'Église comme corps hiérarchique qui est ainsi dessinée, et il est alors patent que des schémas ecclésiologiques concurrents coexistent.

Le dossier présente en outre la particularité d'intégrer la minorité juive au thème général, conformément au souhait, formulé dès 2001 par la revue Médiévales, de «voir l'histoire des juifs traitée avec celle des chrétiens » dans le cadre « d'une histoire globale de la société médiévale ${ }^{6}$, en tenant compte des « interactions » comme des « écarts ${ }^{7}$. Les juifs apparaissent ainsi dans trois des cinq textes du dossier. Des intérêts scientifiques convergents semblent toucher ici un point fondamental :

5. $C f$. C. Avignon, « L'Église et les infractions au lien matrimonial : mariages clandestins et clandestinité. Théories, pratiques et discours. France du Nord-Ouest, XII-milieu du XVI ${ }^{e}$ siècle » (thèse de doctorat sous la direction de L. Feller, université de Paris-Est, 2008, dactylographiée) ; V. BEAULANDE-BARRAUD, « Les cas réservés en Occident, $\mathrm{XIII}^{\mathrm{e}}$-XV ${ }^{\mathrm{e}}$ siècles. Juridiction épiscopale, hiérarchie ecclésiale et pénitence » (mémoire inédit d'HDR soutenu le 5 décembre 2015 à l'Université de Paris-Ouest-Nanterre-La Défense); E. Marmursztejn, Le Baptême forcé des enfants juifs. Question scolastique, enjeu politique, échos contemporains, Paris, 2016 ; Y. MASSET, «Les juifs face à la justice catalane (1301-1327) », thèse de doctorat soutenue le 12 décembre 2015 à l'Université de Nantes; C. Nemo-Perelman, Rome et ses citoyens juifs. $I^{e}-V^{e}$ siècles, Paris, 2010.

6. B. Grévin, D. Iogna-Prat, D. Sansy, « Destin des juifs d'Europe du Nord : une question d'histoire globale », Médiévales, 41 (2001), p. 8.

7. Ibid.,p. 9. 
l'opposition entre Loi ancienne et Loi nouvelle forme le prototype même du conflit de normes dans les sociétés chrétiennes occidentales. Toutefois, ce motif reste ici - au mieux - sous-jacent. Les contributions traitent moins de «juifs théologiques » ou de « juifs herméneutiques » que de « juifs réels ${ }^{8}$. C'est ainsi sous l'angle des conflits de lois et des conflits de juridictions que Capucine Nemo-Pekelman aborde le statut du judaïsme dans l'ordre juridique romain des $\mathrm{IV}^{\mathrm{e}}$ et $\mathrm{v}^{\mathrm{e}}$ siècles. Il s'agit plus précisément « d'expliciter les logiques juridiques qui [...] permirent l'insertion ou le rejet du droit et des instances de jugement juives dans l'ordre juridique romain ». Dans un contexte très différent - celui de la Catalogne du début du XIV ${ }^{\mathrm{e}}$ siècle -, la contribution de Youna Masset recoupe partiellement cette perspective en analysant les conflits de compétence en matière de juridiction sur les juifs, qui opposèrent le roi à l'Église, aux seigneurs laïcs, mais aussi aux autorités juives. Dans le domaine théologique même, Elsa Marmursztejn observe que, contre toute attente, la question du baptême forcé des enfants juifs ne s'inscrit pas sur le terrain « légal » de l'opposition entre judaïsme et christianisme - terrain complexe et dense, où les réflexions théologiques sur l'évacuation des prescriptions cérémonielles vétérotestamentaires se combinent avec la revendication évangélique d'accomplir, et non d'abolir la Loi -, mais sur le terrain politique et juridique de l'opposition entre pouvoir du prince chrétien et droit des parents juifs.

Les conflits entre instances normatives prennent corps dans les contextes spécifiques explorés par les différentes contributrices : conflits entre tribunaux juifs et juridiction impériale aux IV et $v^{\mathrm{e}}$ siècles ; conflits de compétence en matière de juridiction sur les juifs dans la Catalogne du $\mathrm{XIV}^{\mathrm{e}}$ siècle ; divergences théologiques dans le contexte universitaire des XIII ${ }^{\mathrm{e}}$ et XIV ${ }^{\mathrm{e}}$ siècles ; tension entre droit canonique et théologie sur le traitement des mariages «illicites »; concurrence entre la plenitudo potestatis pontificale et le ministère épiscopal, mais aussi entre celui-ci et le "pouvoir des clefs » donné à tous les prêtres par l'ordination. Des instances inhabituelles entrent parfois en jeu : les « tribunaux juifs », qui relèvent du cadre communautaire et le plus souvent de l'institution synagogale, tiennent ce rôle face aux tribunaux publics romains, même s'il est difficile de parler dans ce cas de « conflit de juridictions », ce dernier terme étant réservé, comme le rappelle Capucine Nemo-Pekelman, aux instances de jugement publiques. Dans le même ordre d'idées, les théologiens impliqués dans les controverses scolastiques peuvent être considérés comme des instances normatives, dès lors que l'on reconnait à leur production intellectuelle le caractère normatif que leur confèrent à la fois la forme de la quaestio (proche de celle du casus juridique) et la teneur des solutions que les maitres construisent en

8. Sur cette distinction, on verra notamment G. DaHAN, Les Intellectuels chrétiens et les juifs au Moyen Âge, Paris, 1990 ; et J. CoHEN, Living Letters of the Law. Ideas of the Jew in Medieval Christianity, Berkeley/Los Angeles/Londres, 1999. 
s'appuyant largement sur des autorités juridiques, dans un travail complexe de captation et de mise à distance ${ }^{9}$.

Du reste, les conflits entre instances normatives ne concernent pas toujours des institutions ou des collectivités. La considération de ces conflits ménage les singularités. Si, dans la Catalogne de la fin du Moyen Âge, le roi, d'une part, l'Église, les seigneurs laïcs et les aljamas juives, d'autre part, sont les protagonistes des conflits de compétence en matière de juridiction sur les juifs, ces conflits opposèrent aussi, occasionnellement, les officiers royaux entre eux, au point que le roi doive intervenir à plusieurs reprises, entre le milieu du XIII ${ }^{\mathrm{e}}$ et le début du XIV ${ }^{\mathrm{e}}$ siècle, pour définir les compétences respectives du bayle et du viguier à Barcelone. Dans un registre différent, les divergences théologiques sur la question du baptême forcé des enfants juifs n'ont rien d'artificiel et font pièce aux préjugés du conformisme doctrinal ou du psittacisme imposé par la tradition. La réflexion sur l'exercice de jurisdictio au for de la pénitence ne dessine pas des blocs univoques, entre juristes pontificaux, prélats et Mendiants.

Des conflits, parfois imprévus, opposent et traversent aussi les sources normatives. Constitutions impériales romaines, droit juif, théologie, droit canonique, sommes de pénitence, manuels de confesseurs et statuts synodaux permettent de faire varier les termes des conflits de normes et d'en apprécier la productivité. Dans l'Antiquité tardive, les cas de conflits entre droit romain et lois juives peuvent aussi bien se solder par la censure que par la reconnaissance de ces lois. Si les conflits de compétence ont abouti à refuser toute autonomie juridictionnelle aux instances de jugement juives au niveau du village, de la communauté ou de la synagogue -, puisqu'elles n'acquièrent que le statut d'instances d'arbitrage privées, l'analyse des conflits de lois révèle la façon dont la chancellerie impériale romaine a pu reconnaître dans la loi juive une source de droit, quoiqu'elle l'ait investie d'une moindre auctoritas. Dans le débat scolastique sur le baptême forcé des enfants juifs, la coutume de l'Église ou la patria potestas du père juif se heurtent à la norme universelle du baptême. La licéité de l'administration du sacrement aux enfants des juifs est déduite de la confrontation entre les droits du prince chrétien et des parents juifs. La distinction entre ordre et juridiction au sein du « pouvoir des clefs » résulte, entre autres, des tensions entre for de la conscience et for judiciaire, aboutissant notamment à ce paradoxe déjà évoqué du mariage clandestin peccamineux mais valide.

Enfin, ces conflits de normes ont donné lieu à des résultats variés. À l'époque tardo-antique, les conflits entre lois romaines et lois juives se sont résolus selon un mode substantiel, c'est-à-dire en donnant lieu à la création de nouvelles lois ou de privilèges. En Catalogne, le pouvoir monarchique

9. $C f$. E. Marmursztejn, «Une fabrique de la norme au XiII ${ }^{\mathrm{e}}$ siècle : l'université de Paris », dans V. Beaulande-Barraud, J. Claustre et E. Marmursztejn éd., La Fabrique de la norme..., p. 37-42. 
s'est affirmé, à partir du milieu du XIII ${ }^{\mathrm{e}}$ siècle, en parvenant à étendre sa compétence juridictionnelle face à une large concurrence. Dans la théologie scolastique des $\mathrm{XIII}^{\mathrm{e}}$ et $\mathrm{XIV}^{\mathrm{e}}$ siècles, le choc entre le droit du prince chrétien et les droits des juifs comme parents, comme sujets ou comme esclaves, a donné lieu à des normes divergentes sur le baptême forcé des enfants juifs. Les débats et conflits pratiques autour des cas réservés aboutissent à des compromis pragmatiques, à une répartition des rôles cependant toujours fragile, notamment devant la norme irréductible de la miséricorde de l'Église in articulo mortis. En matière matrimoniale, la doctrine consensualiste impose la validité des mariages clandestins, mais ceux-ci, entachés d'imperfection morale, sont progressivement criminalisés.

Cette variété de situations, qui répond à la diversité des champs de recherche, n'en produit pas moins certains échos, voire certaines cohérences. Ainsi, les conflits de compétence - que ce soit dans l'ordre juridique romain de l'époque postclassique ou entre les protagonistes multiples des conflits catalans du XIV ${ }^{e}$ siècle - donnent lieu à la création de normes nouvelles par le législateur souverain. Les tensions normatives autour du mariage s'inscrivent dans le contexte de la définition très progressive des fors dans les derniers siècles médiévaux, dont le principe de la réserve au for pénitentiel (qu'il soit sacramentel ou judiciaire) témoigne. Dans un autre registre, le motif de la servitude des juifs, qui fonde la revendication monarchique d'une juridiction exclusive sur les juifs en Catalogne, en particulier sous le règne de Jacques II, est un argument majeur du débat théologique des $\mathrm{XIII}^{\mathrm{e}}$ et $\mathrm{XIV}^{\mathrm{e}}$ siècles sur le baptême forcé des enfants des juifs. L'effectivité du principe de la servitude des juifs dans la Catalogne du $X^{\mathrm{e}}$ siècle suggère qu'il ne s'est pas cantonné dans l'abstraction théologique et qu'il participe largement de l'affirmation de la souveraineté chrétienne à la fin du Moyen Âge. Du reste, les penseurs scolastiques ont exprimé cette servitude en termes strictement juridiques et l'ont conçue comme un statut emportant des effets juridiques réels. Le rapprochement entre des conflits de compétences ou des conflits de normes a priori très distants, dont le statut Iudei nostri représente l'un des termes, révèle que ce statut, jamais formalisé, fait pourtant partie d'un système de références partagées, et contribue à éclairer les deux situations, théologique et politique. Enfin, les rôles d'acteurs attribués à des candidats inhabituels contribuent paradoxalement à la cohérence des différentes contributions. Les juifs sont ainsi des acteurs réels des conflits de compétence dans l'Antiquité tardive comme dans la Catalogne de la fin du Moyen Âge. Ils s'y adonnent également, en tant que minorité, au forum shopping. Les théologiens font également figure d'acteurs singuliers. La pratique individuelle de la méthode scolastique, fondée sur la confrontation des autorités discordantes, peut en effet être considérée, en deçà de la controverse proprement dite, comme le premier niveau de pertinence du conflit de normes dans le champ 
scolastique. Les évêques, en matière pénitentielle comme matrimoniale, ne sont pas les simples relais des normes canoniques élaborées dans les écoles et/ou dans l'entourage pontifical, mais bien des acteurs de conflits normatifs résolus de manière pragmatique plus encore que doctrinale.

On voudrait noter, en dernier lieu, que les conflits et les concurrences de normes ne sont pas des jeux purement formels, situés en des sphères abstraites. On l'a dit, le point de vue des acteurs importe, et la considération de leurs positionnements ou de leurs usages stratégiques ne doit pas porter à négliger l'inégalité des rapports de forces, non plus que les conséquences sociales concrètes des conflits de normes dans lesquels ils sont engagés. Outre les modes de résolution, il faut prendre acte de la violence des conflits et de leurs effets. Ainsi, l'invalidité de l'absolution d'un péché réservé par le simple prêtre met en jeu le salut même du fidèle. Lorsqu'un évêque prétend dire quel rite fait ou ne fait pas le mariage, il crée ou ruine un lien fondamental de la société médiévale. Certains théologiens scolastiques ont jugé que les princes chrétiens avaient tout pouvoir sur les enfants de «leurs » juifs. À la fin du IV e siècle, la réduction des juges juifs au rang d'arbitres privés et l'assimilation, par fiction, des tribunaux juifs à des instances d'arbitrage romaines, contredit « l'idée répandue selon laquelle l'Empire romain, contrairement aux États-nations modernes, aurait fourni un cadre juridico-politique exemplaire pour le respect du pluralisme juridique » (Capucine Nemo-Pekelman). Établir la productivité normative des conflits de normes au Moyen Âge, en divers domaines, n'exclut pas de prendre les conflits au sérieux, sans négliger ni édulcorer la substance polémique de ces processus porteurs d'effets juridiques et sociaux. La recherche de ce point d'équilibre est ce qui réunit, plus globalement que les perspectives communes ici esquissées, les différents travaux présentés dans ce dossier.

Véronique Beaulande-Barraud - Université de Reims - CERHIC EA 2616

Elsa Marmursztejn - Université de Reims - CERHIC EA 2616 
